\title{
Cost-Effectiveness of Vedolizumab in the Treatment of Moderate-to-Severe Crohn's Disease in China
}

Ting Zhou · Yanan Sheng $\cdot$ Haijing Guan

Received: April 30, 2021 / Accepted: May 24, 2021 / Published online: June 5, 2021

(C) The Author(s) 2021

\begin{abstract}
Introduction: To compare the cost-effectiveness of vedolizumab with that of conventional therapy in patients with moderate-to-severe active Crohn's disease (CD) in China.

Methods: A decision tree and Markov model were built to predict the lifetime cost and health outcomes in the induction phase and maintenance phase of vedolizumab treatment and conventional therapy (a combination of corticosteroids, immunosuppressants, and aminosalicylates) in adult patients with moderate-tosevere active $\mathrm{CD}$ from the perspective of China's healthcare system. Clinical efficacy and health utility were derived from the GEMINI 2 and
\end{abstract}

Supplementary Information The online version contains supplementary material available at https:// doi.org/10.1007/s12325-021-01806-7.

\section{T. Zhou}

School of International Pharmaceutical Business, China Pharmaceutical University, Nanjing, China

\section{Y. Sheng}

Medical Affairs, Takeda (China) International

Trading Company, Beijing, China

H. Guan $(\bowtie)$

Department of Pharmacy, Beijing Tiantan Hospital, Capital Medical University, Beijing, China

e-mail: guanhaijing_1@163.com

H. Guan

China Center for Health Economic Research, Peking University, Beijing, China
GEMINI 3 trials and published literature. Costs were mainly obtained from clinical physician surveys in China and are presented in 2020 US dollars. Health outcomes (quality-adjusted life years, QALYs) and costs were discounted at an annual rate of $5 \%$. The incremental cost per QALY gained was used to compare the cost-effectiveness of the two treatments. One-way and probabilistic sensitivity analyses (PSAs) were performed to test the robustness of the model. Results: The model predicted more QALYs (9.92 vs 9.00 QALYs) and lower incurred costs $(\$ 288,284$ vs $\$ 309,680)$ in vedolizumab than in conventional therapy in a mixed population (anti-TNF-naïve and anti-TNF-failure populations) over a lifetime horizon in the base-case analysis. Similar results were observed in the anti-TNF-naïve and anti-TNF-failure subgroups of patients with CD. One-way sensitivity analysis results suggested that health state cost was the most influential factor in the model. The PSA results supported the dominance of vedolizumab in the base-case analysis.

Conclusion: Vedolizumab appears to be a costeffective strategy option in the treatment of adult patients with moderate-to-severe active CD in China in both anti-TNF-naïve and antiTNF-failure populations.

Keywords: China; Conventional therapy; Costeffectiveness analysis; Crohn's disease; Vedolizumab 


\section{Key Summary Points}

In recent years, cost-effectiveness analysis has been applied to determine entry onto the National Reimbursement Drug List in China. With the limited alternative drugs for the treatment of Crohn's disease (CD), more than $90 \%$ of patients are treated with conventional therapy drugs. As a new-generation biological drug, vedolizumab (VDZ) was approved to treat CD in March 2020 in China and it provided a new option for the patients.

Economic evaluation evidence of vedolizumab is needed to support health decision-making in China. A hybrid decision tree and Markov model were first developed to compare the lifetime costs and health outcomes of VDZ and CT in the treatment of Chinese patients with moderate-to-severe active CD using published efficacy data of GEMINI 2 and GEMINI 3 trials in China.

Compared with CT, VDZ appears to be a cost-effective option in the treatment of adult patients with moderate-to-severe active CD in China in both anti-TNFnaïve and anti-TNF-failure populations.

\section{DIGITAL FEATURES}

This article is published with digital features, including a summary slide, to facilitate understanding of the article. To view digital features for this article, go to https://doi.org/10.6084/ m9.figshare.14627499.

\section{INTRODUCTION}

Crohn's disease (CD) is a chronic progressive and relapsing inflammatory bowel disease (IBD) that affects the gastrointestinal tract and leads to bowel damage and disability with the development of disease [1]. The incidence and prevalence of $\mathrm{CD}$ have increased worldwide, especially in developed countries [2]. The incidence rate varies from 16.7 to 318.5 per 100,000 persons in North America, while it varies from 0.88 to 67.9 per 100,000 persons in Asia and the Middle East [2]. China is one of the developing countries facing a steady increase in patients with $\mathrm{CD}$, and the incidence rate ranges from 0.07 to 1.31 per 100,000 persons [3]. Patients with CD suffer from a heavy disease burden, poor quality of life, and financial problems [4]. It is estimated that the direct costs of CD vary from $\$ 18,022$ to $\$ 18,932$ per patient-year in the USA and from $€ 2898$ to $€ 6960$ in European countries $[5,6]$. In China, with the increasing usage of healthcare resources for IBD treatment, the mean direct cost rose to $\$ 11,669$ per patientyear in 2019 [7].

Under current medical technology conditions, CD is an incurable disease. The treatment of CD mainly focuses on relieving symptoms and achieving long-term remission [1]. For conventional therapy (CT), corticosteroids, immunosuppressants and aminosalicylates are used to treat CD in most instances [1]. In China, more than $90 \%$ of patients are treated with CT drugs [8]. Compared with CT, biological regimens (e.g., anti-TNF $\alpha$ agents, anti-integrins) are effective in patients with $\mathrm{CD}$ in achieving clinical response and remission and are treatment alternatives, especially for patients who fail conventional treatment $[9,10]$. However, $10-30 \%$ of patients with IBD have no response to initial anti-TNF therapy, and over $23 \%$ of patients with IBD lose response during the course of treatment [11]. As a new-generation biological drug, vedolizumab is an intravenously administered humanized immunoglobulin G1 monoclonal antibody that blocks $\alpha 4 \beta 7$ integrin and modulates the gut lymphocyte trafficking [1]. The post hoc analysis results of the GEMINI 2 and GEMINI 3 trials suggest that vedolizumab is effective for both anti-TNF-naïve and anti-TNF-failure patients with CD [12].

Economic evaluations play an important role in health decision-making and drug reimbursement in China [13]. Vedolizumab was approved by China's National Medical Products Administration (NMPA) in adults with moderate-to- 
severe active CD in March 2020 and is covered by Chinese national basic insurance. This study aimed to compare the cost-effectiveness of vedolizumab with that of $\mathrm{CT}$ in patients with moderate-to-severe active CD in China.

\section{METHODS}

\section{Model Description}

The model developed to perform cost-effectiveness analysis in this study was based on a previous model submitted to the National Institute for Health and Care Excellence (NICE) and subsequently revised [14]. This model consists of a decision tree (Fig. 1a) to simulate shortterm induction therapy and a lifetime Markov model (Fig. 1b) to simulate maintenance therapy. Five health states are structured, three of which are defined by the Crohn's Disease Activity Index (CDAI) score: remission (CDAI score less than 150), mild (CDAI score 150-220), and moderate-to-severe (CDAI score 220-600). The other two health states are surgery and death. During the induction phase, adults with moderate-to-severe active $\mathrm{CD}$ entered the model and initiated treatment with vedolizumab or CT. Patients who used biological therapy were monitored for response to the drug at the end of a 6-week induction phase, which was consistent with the duration in clinical trials $[15,16]$.

Patients on vedolizumab treatment who responded to therapy and did not discontinue because of adverse event intolerability entered

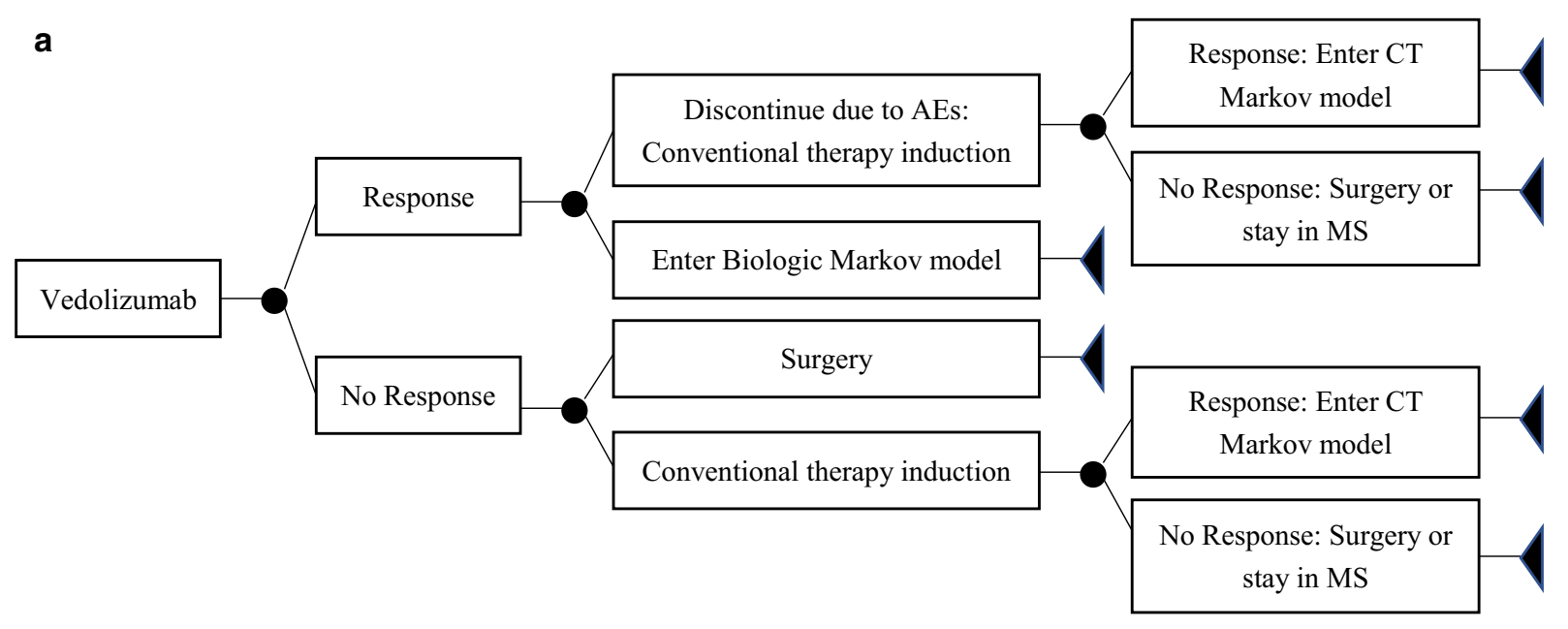

b

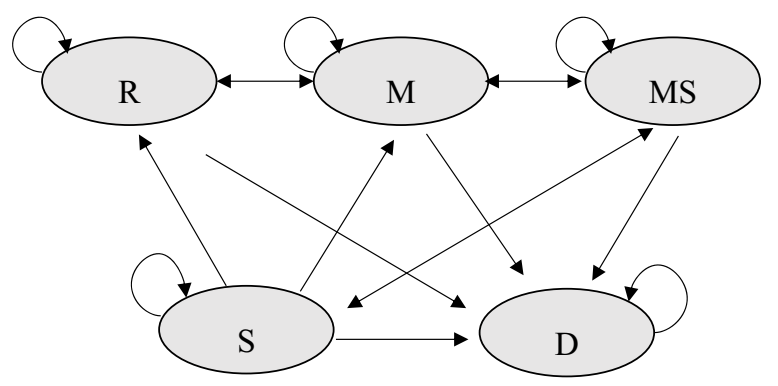

Fig. 1 a Decision tree framework of induction phase. AEs adverse events, CT conventional therapy, MS moderate-tosevere, CDAI Crohn's Disease Activity Index. Response is defined as reduction in the CDAI score of at least 70 points or remission (CDAI score less than 150 points). b Markov model underlying the maintenance phase. $\mathrm{R}$ remission, $\mathrm{M}$ mild, MS moderate-to-severe, $\mathrm{S}$ surgery, $\mathrm{D}$ death 
the biological Markov model with 8-week cycles for maintenance therapy. In the Markov model, patients could transition among each of the four health states (remission, mild, moderateto-severe, and surgery), transition to death, or remained in the same state at the end of each cycle. Patients in the moderate-to-severe health state after 1 year on vedolizumab treatment would discontinue because of a lack of response and switched to CT or surgery. Patents who discontinued because of adverse events (assumed to occur only in the biological arm) were assumed to switch to CT in the maintenance phase. In the CT arm, patients who responded in induction phase entered the Markov model for CT. Otherwise, patients were assumed to stay in a moderate-to-severe state until they required surgery if they failed to respond to induction treatment.

From China's healthcare system perspective, this model simulated the disease progression of adult patients with CD over a lifetime horizon. Costs were adjusted to 2020 US dollar currency. Health outcomes (quality-adjusted life years, QALYs) and costs were discounted annually at $5 \%$ in line with the recommendation of Chinese guidelines for pharmacoeconomic evaluation [17].

\section{Model Inputs and Data Sources}

\section{Patient Characteristics}

The model focused on adult patients with moderate-to-severe active CD in China, which was defined as having CDAI scores over 220. The mean age of the patients was 37.3 years, and $55.4 \%$ were male, with an average weight of $50.83 \mathrm{~kg}$ [3]. A hypothetical mixed population cohort of 1000 anti-TNF-naïve and anti-TNFfailure patients were included in the simulation. Each subgroup of the mixed population was analyzed individually.

\section{Treatments}

In the biological arm, vedolizumab was given in 300-mg intravenous infusions at weeks 0, 2 and 6 (if had response) in the induction phase and every 8 weeks in the maintenance phase. In the CT arm, a combination of corticosteroids, immunosuppressants, and aminosalicylates was given (including mesalazine, prednisolone, azathioprine, sulfasalazine, budesonide, balsalazide, olsalazine, methotrexate, etc.), which was in line with the recommendation in Chinese guidelines for patients with CD [18].

\section{Clinical Efficacy and Transition Probability}

Clinical efficacy included the response and remission rate for the induction phase and the probability of staying in remission or mild disease during the maintenance phase. Response was defined as a decrease in the CDAI score of more than 70 points, while remission was defined as the CDAI score of 150 or less. The efficacy data of response and remission in each group were derived from two randomized clinical trials (RCTs) (GEMINI 2 and GEMINI 3) of comparisons of vedolizumab with CT plus placebo biological therapy $[15,16]$. The probability of surgery was based on published literature $[19,20]$. The transition probabilities for health states in each cycle are presented in Tables 1-3 in supplementary material.

\section{Adverse Events and Discontinuation}

The probabilities of adverse events and discontinuation were based on clinical trials $[15,16]$ and are presented in Table 1. In this model, discontinuation occurred when there was a lack of response to induction treatment, a loss of response in the maintenance phase, and adverse event intolerability in the vedolizumab arm $[15,16]$.

\section{Mortality}

Age- and sex-specific all-cause mortality was obtained from the Chinese general population [21]. Mortality was adjusted according to the patients' baseline in this model, and an exponential distribution was estimated to predict mortality in each cycle with increasing age. The health-state-specific relative risk (RR) of mortality for each health state was estimated from published literature [22]. Compared with remission, the RRs of mortality for mild, moderate-to-severe, and surgery were 1.27, 2.26, and 3.22 , respectively, with the progression of disease [22]. 
Table 1 Model inputs of clinical efficacy, adverse events, and discontinuation

\begin{tabular}{|c|c|c|c|c|c|c|c|}
\hline & \multicolumn{3}{|c|}{ Vedolizumab } & \multicolumn{3}{|l|}{ CT } & \multirow[t]{2}{*}{ References } \\
\hline & Mixed & $\begin{array}{l}\text { Anti-TNF } \\
\text { naïve }\end{array}$ & $\begin{array}{l}\text { Anti-TNF } \\
\text { failure }\end{array}$ & Mixed & $\begin{array}{l}\text { Anti-TNF } \\
\text { naïve }\end{array}$ & $\begin{array}{l}\text { Anti-TNF } \\
\text { failure }\end{array}$ & \\
\hline \multicolumn{8}{|l|}{ Clinical efficacy } \\
\hline \multicolumn{8}{|c|}{ At the end of the induction phase } \\
\hline $\begin{array}{l}\text { 6-week probability of } \\
\text { response }\end{array}$ & $48.02 \%$ & $53.75 \%$ & $45.25 \%$ & $33.80 \%$ & $38.71 \%$ & $30.84 \%$ & {$[15,16]$} \\
\hline $\begin{array}{l}\text { 6-week probability of } \\
\text { remission }\end{array}$ & $16.78 \%$ & $21.88 \%$ & $13.31 \%$ & $9.86 \%$ & $10.32 \%$ & $9.69 \%$ & {$[15,16]$} \\
\hline \multicolumn{8}{|c|}{ During the maintenance phase } \\
\hline $\begin{array}{l}\text { Annual probability of } \\
\text { response }\end{array}$ & $47.40 \%$ & $65.15 \%$ & $29.27 \%$ & $35.29 \%$ & $42.25 \%$ & $26.92 \%$ & {$[15,16]$} \\
\hline $\begin{array}{l}\text { Annual probability of } \\
\text { remission }\end{array}$ & $38.96 \%$ & $51.52 \%$ & $28.05 \%$ & $21.57 \%$ & $26.76 \%$ & $12.82 \%$ & {$[15,16]$} \\
\hline \multicolumn{8}{|l|}{ Incidence of AEs } \\
\hline Serious infection & $0.69 \%$ & & & $1.49 \%$ & & & {$[15,16]$} \\
\hline Tuberculosis & $0.00 \%$ & & & $0.00 \%$ & & & {$[15,16]$} \\
\hline $\begin{array}{l}\text { Malignancy (including } \\
\text { lymphoma) }\end{array}$ & $0.00 \%$ & & & $0.05 \%$ & & & {$[15,16]$} \\
\hline $\begin{array}{l}\text { Acute hypersensitivity } \\
\text { reactions }\end{array}$ & $0.00 \%$ & & & $0.00 \%$ & & & {$[15,16]$} \\
\hline Skin site reactions & $0.93 \%$ & & & $4.50 \%$ & & & {$[15,16]$} \\
\hline $\begin{array}{l}\text { Probability of } \\
\text { discontinuation }^{\mathrm{a}}\end{array}$ & & & & - & & & {$[15,16]$} \\
\hline Induction phase & $9.01 \%$ & $13.23 \%$ & $5.68 \%$ & - & & & {$[15,16]$} \\
\hline $\begin{array}{l}\text { Maintenance phase per } \\
\text { cycle }\end{array}$ & $34.65 \%$ & $15.49 \%$ & $68.29 \%$ & - & & & {$[15,16]$} \\
\hline
\end{tabular}

$V D Z$ Vedolizumab, $C T$ conventional therapy, $A E$ adverse event

${ }^{a}$ Discontinuation due to AEs is applicable only to responders on biological treatments because non-responders on biologics switch to CT and continue receiving such until the end of the model's time horizon or until the patients require surgery

\section{Health State Utility and Disutility}

The health state utility and disutility applied in this model were systematically identified from published sources (Table 2) [23-29]. QALYs were calculated by using utility values multiplying by the duration in the health state.

\section{Costs}

Drug acquisition and administration costs, health state costs, and adverse event management costs were included in this model (Table 2). The price of vedolizumab was the shortlisted price of China's National Reimbursement Drug List in 2020 [30]. The combination of treatments for CT was based on a 
Table 2 Model inputs of costs and health utility

\begin{tabular}{|c|c|c|c|}
\hline & Costs $(\$)$ & Utility & References \\
\hline \multicolumn{4}{|l|}{ Drug cost per cycle } \\
\hline Vedolizumab induction phase & $1539.4^{\mathrm{b}}$ & - & {$[30]$} \\
\hline Vedolizumab maintenance phase & 769.7 & - & {$[30]$} \\
\hline Conventional therapy $^{\mathrm{a}}$ & 362.5 & - & Expert survey \\
\hline Administration cost per cycle ${ }^{a}$ & 50.7 & - & Expert survey \\
\hline Health state costs per cycle ${ }^{a}$ & & Health state utility & \\
\hline Remission & 386.8 & 0.83 & {$[23]$} \\
\hline Mild & 440.9 & 0.69 & {$[23]$} \\
\hline Moderate-to-severe & 1949.9 & 0.42 & {$[23]$} \\
\hline Surgery & $10,601.8$ & 0.42 & {$[23,24]$} \\
\hline Costs of AEs per event ${ }^{a}$ & & AE utility decrement & \\
\hline Serious infection & 1504.2 & -0.47 & {$[25]$} \\
\hline Tuberculosis & 815.3 & -0.50 & {$[26]$} \\
\hline Malignancy (including lymphoma) & 1507.4 & -0.18 & {$[27]$} \\
\hline Acute hypersensitivity reactions & 234.0 & -0.10 & {$[28]$} \\
\hline Skin site reactions & 46.8 & -0.03 & [29] \\
\hline
\end{tabular}

$A E$ adverse event

${ }^{a}$ The price information is based on the survey of 18 clinical physicians from 18 tertiary hospitals in China. ${ }^{\mathrm{b}}$ The cost was for weeks 0 and 2. A third injection was given if had response at week 6

clinical physician survey of 18 doctors in 18 tertiary hospitals in China in 2019, as well as the related costs, summarized in Tables 4 and 5 in the supplementary material. Mesalazine (46\%) was the most commonly used drug in CT, followed by prednisolone (41\%), and the weighted cost was $\$ 362.5$ per cycle in China. In addition, health state costs, administration fees, and adverse event treatment costs were derived from the clinical expert survey mentioned above.

\section{Cost-Effectiveness Analysis}

In the base-case analysis, lifetime discounted costs and health outcomes were calculated for biological treatment and CT in a mixed population (anti-TNF-naïve and anti-TNF-failure patients). The incremental cost per QALY gained was compared with the willingness-topay threshold in China, three times the Chinese GDP per capita in $2020(\$ 31,500)$. Values for the subgroups of anti-TNF-naïve and anti-TNF-failure patients were estimated.

One-way sensitivity analysis and probabilistic sensitivity analysis (PSA) were performed to validate the robustness of specific parameter estimates, given their uncertainty. Variation in the parameter values of one-way sensitivity analysis was based on the 95\% confidence interval or $\pm 20 \%$ when such data were not available. PSA was carried out by 5000 Monte Carlo simulations varying all input parameters at the same time on the basis of prespecified distributions. Cost and mortality RR, rate and utility, and transition probability parameters were assumed to follow gamma, beta, and Dirichlet distributions. 
This article is based on previously conducted studies and does not contain any new studies with human participants or animals performed by any of the authors. No patient identifiable data were involved in the analysis. Therefore, institutional review board approval was not required.

\section{RESULTS}

\section{Base-Case Analysis}

The results of the base-case analysis that compared vedolizumab and CT in patients with CD over a lifetime horizon are presented in Table 3. For the mixed (anti-TNF-naive and anti-TNFfailure patients) population, treatment with vedolizumab resulted in 0.92 more QALYs (9.92 vs 9.00 QALYs) gained but lower discounted lifetime costs $(\$ 288,284$ vs $\$ 309,680)$ than treatment with $\mathrm{CT}$. In addition, vedolizumab therapy led to a higher QALY gained per patient (1.40 vs 0.58 QALYs) and a lower incremental cost $(-\$ 33,484$ vs $-\$ 13,076)$ in the anti-TNFnaïve population than in the anti-TNF-failure population compared with CT. Therefore, biological treatment with vedolizumab appears to be a dominant strategy in patients with moderate-to-severe CD.

\section{Sensitivity Analysis}

The results of one-way sensitivity analyses are shown in Fig. 2. The incremental cost per QALY gained for vedolizumab was most sensitive to the health state costs, followed by average starting age entered in the model, for anti-TNFnaïve patients, anti-TNF-failure patients, and the mixed population. In addition, the one-way sensitivity analysis results were in line with the base-case analysis results; compared with CT, vedolizumab seemed to be a dominant strategy when the parameters varied with the estimated range.

The PSA results were robust to changes in all parameters at the same time following the predefined distribution and assumptions (Fig. 3). In the mixed population, vedolizumab was costeffective in $100 \%$ of simulations and dominant in over $99 \%$ of all simulations (fourth quadrant) compared with CT. Similar PSA results were

Table 3 Results of base-case analyses

\begin{tabular}{llll}
\hline & Vedolizumab & Conventional therapy & Difference \\
\hline Mixed population & & & $-21,397$ \\
Costs & 288,284 & 309,680 & 0.92 \\
QALYs & 9.92 & 9.00 & Dominant \\
ICER (\$ per QALY gained) & & & \\
Anti-TNF-naïve population & & & $-33,484$ \\
Costs & 264,301 & 297,785 & 1.40 \\
QALYs & 10.86 & 9.46 & Dominant \\
ICER (\$ per QALY gained) & & & $-13,076$ \\
Anti-TNF-failure population & & & 0.58 \\
Costs & 303,551 & 316,627 & Dominant \\
QALYs & 9.32 & 8.74 & \\
ICER (\$ per QALY gained) & & &
\end{tabular}

ICER incremental cost-effectiveness ratio 


\section{a}

Health State Costs(-/+20\%)

Starting age of population $(95 \% \mathrm{CI})$

CT transition probabilities: moderate-severe $(95 \% \mathrm{CI})$

Health state utilities (+/- 20\%)

CT transition probabilities: mild $(95 \% \mathrm{CI})$

Relative risk of mortality by health state $(+/-20 \%)$

CT induction response/remission $(95 \% \mathrm{CI})$

CT transition probabilities: remission $(95 \% \mathrm{CI})$

Percentage of patients requiring surgery $(95 \% \mathrm{CI})$

VDZ transition probabilities: moderate-severe $(95 \% \mathrm{CI})$

VDZ discontinuation rate $(95 \% \mathrm{CI})$

Percentage of non-remitting responders in $\mathrm{M} / \mathrm{S}(95 \% \mathrm{CI})$

AE Incidence- Conventional therapy $(95 \% \mathrm{CI})$

VDZ transition probabilities: mild (95\% CI)

VDZ induction response/remission $(95 \% \mathrm{CI})$

$$
\$-32,000
$$

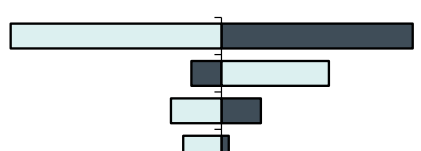

1

I

4

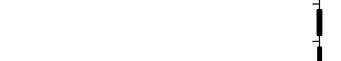

b

Health State Costs(-/+20\%)

Starting age of population $(95 \% \mathrm{CI})$

CT transition probabilities: moderate-severe $(95 \% \mathrm{CI})$

CT transition probabilities: remission $(95 \% \mathrm{CI})$

CT transition probabilities: mild (95\% CI)

Health state utilities (+/- 20\%)

Relative risk of mortality by health state $(+/-20 \%)$

CT induction response/remission $(95 \% \mathrm{CI})$

Percentage of patients requiring surgery $(95 \% \mathrm{CI})$

VDZ transition probabilities: moderate-severe (95\% CI)

VDZ induction response/remission $(95 \% \mathrm{CI})$

AE Incidence- Conventional therapy (95\% CI)

VDZ transition probabilities: mild (95\% CI)

VDZ discontinuation rate $(95 \% \mathrm{CI})$

VDZ transition probabilities: remission $(95 \% \mathrm{CI})$

$$
\$-32,000
$$

$\$-28,000$

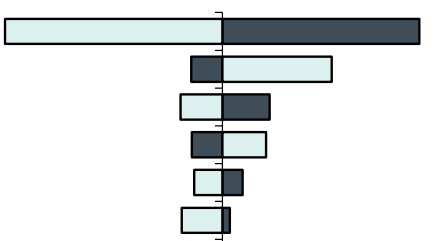

Incremental Cost per QALY Gained
C

Health State Costs(-/+20\%)

Starting age of population $(95 \% \mathrm{CI})$

CT transition probabilities: mild $(95 \% \mathrm{CI})$

CT transition probabilities: moderate-severe $(95 \% \mathrm{CI})$

Health state utilities (+/- 20\%)

CT induction response/remission $(95 \% \mathrm{CI})$

Relative risk of mortality by health state $(+/-20 \%)$

Percentage of non-remitting responders in $\mathrm{M} / \mathrm{S}(95 \% \mathrm{CI})$

Percentage of patients requiring surgery $(95 \% \mathrm{CI})$

VDZ transition probabilities: moderate-severe (95\% CI)

VDZ discontinuation rate $(95 \% \mathrm{CI})$

CT transition probabilities: remission $(95 \% \mathrm{CI})$

AE Incidence- Conventional therapy $(95 \% \mathrm{CI})$

VDZ induction response/remission $(95 \% \mathrm{CI})$

VDZ transition probabilities: mild ( $95 \% \mathrm{CI})$

Incremental Cost per QALY Gained

口Lower Bound

Upper Bound $\$-16,000$ 
4Fig. 2 One-way sensitivity analysis for vedolizumab versus conventional therapy: a mixed population, $\mathbf{b}$ anti-TNFnaïve subgroup, $\mathbf{c}$ anti-TNF-failure subgroup. AE adverse event, CI confidence interval, CT conventional therapy, QALY quality-adjusted life years, VDZ vedolizumab

obtained in the subgroups of anti-TNF-naïve and anti-TNF-failure patients.

\section{DISCUSSION}

To our knowledge, this study was the first costeffectiveness analysis of vedolizumab versus CT in adult patients with moderate-to-severe active $\mathrm{CD}$ in China. The base-case analysis results suggested that vedolizumab was a dominant strategy to treat anti-TNF-naïve, anti-TNF-failure, and mixed patients with $\mathrm{CD}$ in China compared with CT. Health state costs and the average starting age were the most influential factors of cost-effectiveness. The results of oneway sensitivity analyses and PSAs were robust to those of base-case analyses. Compared with conventional therapy, vedolizumab resulted in 0.92 QALYs gained for patients with CD. In other words, it was equivalent to patients living in full health for 0.92 years. From this aspect, the absolute difference was not small and the improvement of health was acceptable. Besides that, more than 10,000 US dollars could be saved, which was roughly equivalent to the GDP per capita in China in 2020.

In this study, the clinical efficacy of response and remission in the induction and maintenance phases were mainly estimated from two RCTs $[15,16]$ (GEMINI 2 and GEMINI 3) comparing vedolizumab and CT plus placebo biological therapy. The GEMINI 2 trial [16] was designed to evaluate clinical efficacy and safety for induction and maintenance treatment, while the GEMINI 3 trial [16] focused only on induction therapy. The higher response and remission rates observed in vedolizumab arms resulted in an improvement in overall survival and more QALYs gained.

Discontinuation was defined as lack of response, a loss of response, or an intolerability of adverse events. In this model, discontinuation was assumed to occur only in biological therapy, while patients on CT would receive treatment until the end of the simulation horizon or transition to the surgery state. High discontinuation rates were observed in the anti-TNF-failure patients in the maintenance phase, reflecting the severity of disease in the subgroup population and the challenges in maintaining response. Approximately $68 \%$ of patients in GEMINI 2 lost response at week 52 [16].

Health state utility was calculated using a linear regression estimated by Buxton and colleagues [23] to map CDAI scores to EQ-5D utility values with the algorithm EQ$5 \mathrm{D}=0.9168-0.0012 \times$ CDAI. The average of the CDAI range for each health state was applied to derive utility. For the surgery state, patients experience a period of decremental quality of life after transitioning from the moderate-to-severe state before the surgical procedure and then progressively improving to the remission or mild state after the surgical procedure for the remainder of the cycle in the maintenance phase. Thus, the surgery state was assumed to have the same utility value as moderate-to-severe disease in the model $[22,23]$.

Two health technology assessment (HTA) reports of vedolizumab in the treatment of adult patients with moderate-to-severe active CD were published by NICE [14] and Scottish Medicines Consortium (SMC) [31]. In the NICE single technology appraisal, the model structure submitted by the company was similar to that in this study. Compared with CT, the incremental cost-effectiveness ratio (ICER) of vedolizumab was $£ 21,620$ per QALY gained within the anti-TNF-failure population, while it was above $£ 30,000$ per QALY gained in the mixed population and anti-TNF-naïve population. However, vedolizumab therapy was a cost-saving strategy when the patient access scheme was proposed in the SMC report, which was in line with our study. Vedolizumab was recommended to be used in anti-TNF-failure populations in NHS England and Scotland [14, 31].

A universal health coverage policy has been applied in China for many years. To improve patient access to high-value drugs, China's 


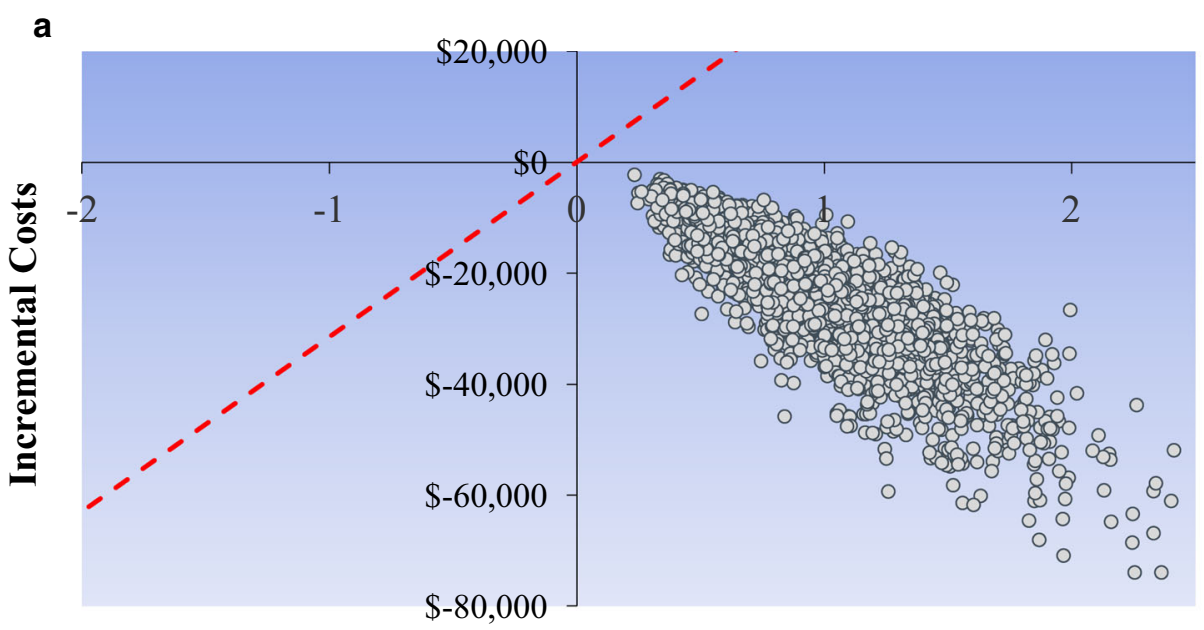

Incremental Quality-adjusted life years (QALYs)

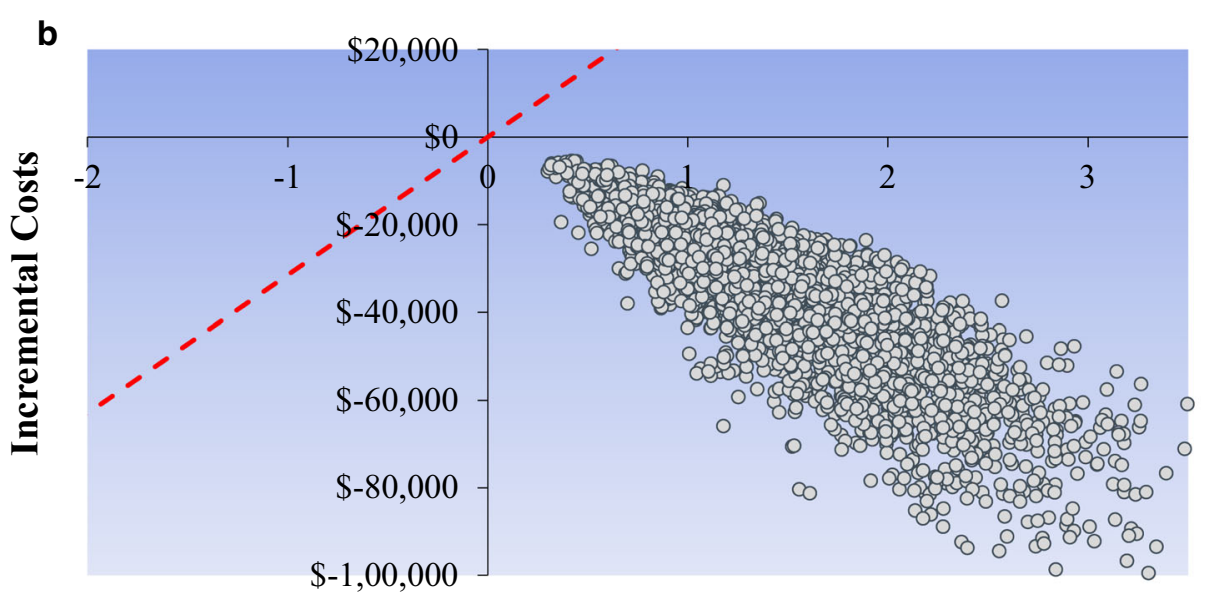

Incremental Quality-adjusted life years (QALYs)

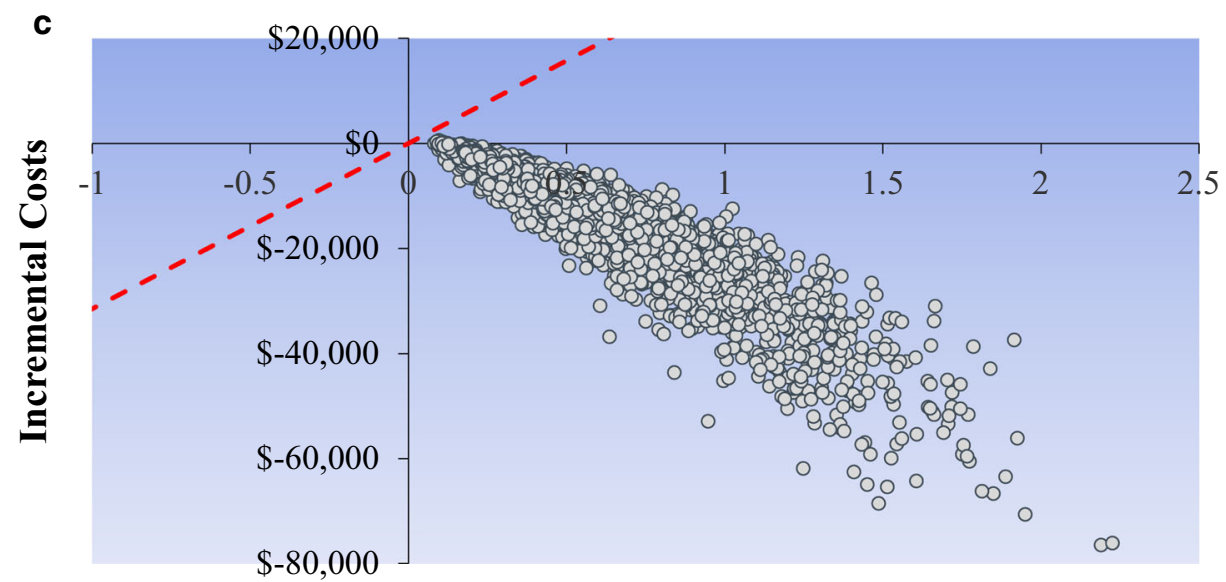

Incremental Quality-adjusted life years (QALYs) 
4Fig. 3 Incremental cost-effectiveness plane of vedolizumab versus conventional therapy: a mixed population, $\mathbf{b}$ antiTNF-naïe subgroup, $\mathbf{c}$ anti-TNF-failure subgroup

National Healthcare Security Administration started price negotiations with pharmaceutical manufacturers in 2017 [13]. To be listed on the National Drug Reimbursement List, the manufacturer of vedolizumab has reached an agreement with the Chinese government to decrease the price by $71 \%$ in 2020 . Therefore, the agreement price of vedolizumab applied in this model from the perspective of China's healthcare system incurred lower costs than those in other countries' settings. In China, vedolizumab is accepted to cover both anti-TNF-naïve and anti-TNF-failure adult patients with moderate-to-severe active $\mathrm{CD}$ by national medical insurance. Moreover, under the current policy in China, renegotiation of the price will be carried out every 2 years by the government. Economic evaluation evidence is needed to support the decision of whether the drug will remain covered by national medical insurance or will be dropped from the reimbursement list in China. As a result of the lack of local economic evaluation information of vedolizumab in the treatment of patients with CD in China, this study is helpful and provides the evidence to inform decision-making.

There are some limitations in this study. Local costs were mainly derived from the clinical physician survey in 18 tertiary hospitals in China, and only direct medical costs were analyzed in the model, which may have impacted the cost-effectiveness, as the results illustrated in the one-way sensitivity analysis. The efficacy data simulated in the model were mainly from RCTs conducted in multiple centers, which may not reflect real clinical practice in China. The results of the sensitivity analysis showed the robustness of the model.

\section{CONCLUSION}

This study suggested that vedolizumab therapy appears to be a cost-effective strategy compared with CT in China. In all subgroups, vedolizumab was cost-effective compared with CT in the treatment of adults with moderate-to-severe active CD in China.

\section{ACKNOWLEDGEMENTS}

Funding. This study analysis was conducted using a model developed by Takeda (China) International Trading Co. Ltd. No additional funding was provided by Takeda (China) International Trading Co. Ltd. The journal's rapid service and open access fees were funded by Ting Zhou and Haijing Guan.

Editorial Assistance. Language editing support was provided by the "Nature research editing service" of Springer Nature. No funding was received for this work.

Authorship. All named authors meet the International Committee of Medical Journal Editors (ICMJE) criteria for authorship for this article, take responsibility for the integrity of the work as a whole, and have given their approval for this version to be published.

Authorship Contributions. TZ, YNS, and HJG designed this study. TZ and HJG collected data and performed analysis. TZ and YNS prepared the manuscript according to HJG's comments. All authors approved the final version of this article.

Disclosures. Yanan Sheng is an employee of Takeda (China) International Trading Co. Ltd. Ting Zhou and Haijing Guan have nothing to disclose.

Compliance with Ethics Guidelines. This article is based on previously conducted studies and does not contain any new studies with human participants or animals performed by any of the authors. All data applied in this study were obtained from published literature, public data and expert surveys. No patient identifiable data were involved in the analysis. Therefore, 
institutional review board approval was not required.

Data Availability. All data generated or analyzed during this study are included in this published article/as supplementary information files.

Open Access. This article is licensed under a Creative Commons Attribution-NonCommercial 4.0 International License, which permits any non-commercial use, sharing, adaptation, distribution and reproduction in any medium or format, as long as you give appropriate credit to the original author(s) and the source, provide a link to the Creative Commons licence, and indicate if changes were made. The images or other third party material in this article are included in the article's Creative Commons licence, unless indicated otherwise in a credit line to the material. If material is not included in the article's Creative Commons licence and your intended use is not permitted by statutory regulation or exceeds the permitted use, you will need to obtain permission directly from the copyright holder. To view a copy of this licence, visit http://creativecommons.org/licenses/by$\mathrm{nc} / 4.0 /$.

\section{REFERENCES}

1. Torres J, Mehandru S, Colombel JF, et al. Crohn's disease. Lancet. 2017;389(10080):1741-55.

2. Molodecky NA, Soon IS, Rabi DM, et al. Increasing incidence and prevalence of the inflammatory bowel diseases with time, based on systematic review. Gastroenterology. 2012;142(1):46-54.

3. Li X, Song P, Li J, et al. The disease burden and clinical characteristics of inflammatory bowel disease in the Chinese population: a systematic review and meta-analysis. Int J Environ Res Public Health. 2017;14(3):238.

4. Knowles SR, Graff LA, Wilding H, et al. Quality of life in inflammatory bowel disease: a systematic review and meta-analyses-part I. Inflamm Bowel Dis. 2018;24(4):742-51.

5. Burisch J, Vardi H, Schwartz D, et al. Health-care costs of inflammatory bowel disease in a pan-
European, community-based, inception cohort during 5 years of follow-up: a population-based study. Lancet Gastroenterol Hepatol. 2020;5(5): 454-64.

6. Yu AP, Cabanilla LA, Wu EQ, et al. The costs of Crohn's disease in the United States and other Western countries: a systematic review. Curr Med Res Opin. 2008;24:319-28.

7. Yu Q, Zhu C, Feng S, et al. Economic burden and health care access for patients with inflammatory bowel diseases in China: web-based survey study. J Med Internet Res. 2021;23(1):e20629.

8. Wei SC. Differences in the public medical insurance systems for inflammatory bowel disease treatment in Asian countries. Intest Res. 2016;14(3):218-23.

9. Danese S, Vuitton L, Peyrin-Biroulet L. Biologic agents for IBD: practical insights. Nat Rev Gastroenterol Hepatol. 2015;12(9):537-45.

10. Pagnini C, Siakavellas SI, Bamias G. Systematic review with network meta-analysis: efficacy of induction therapy with a second biological agent in anti-TNF-experienced Crohn's disease patients. Gastroenterol Res Pract. 2018;2018:6317057.

11. Roda G, Jharap B, Neeraj N, et al. Loss of response to anti-TNFs: definition, epidemiology, and management. Clin Transl Gastroenterol. 2016;7(1):e135.

12. Sands BE, Sandborn WJ, Van Assche G, et al. Vedolizumab as induction and maintenance therapy for Crohn's disease in patients naïve to or who have failed tumor necrosis factor antagonist therapy. Inflamm Bowel Dis. 2017;23(1):97-106.

13. Yue X, Li Y, Wu J, et al. Current development and practice of pharmacoeconomic evaluation guidelines for universal health coverage in China. Value Health Reg Issues. 2020;24:1-5.

14. Rafia R, Scope A, Harnan S, et al. Vedolizumab for treating moderately to severely active Crohn's disease after prior therapy: an evidence review group perspective of a NICE Single Technology Appraisal. Pharmacoeconomics. 2016;34(12):1241-53.

15. Sands BE, Feagan BG, Rutgeerts P, et al. Effects of vedolizumab induction therapy for patients with Crohn's disease in whom tumor necrosis factor antagonist treatment failed. Gastroenterology. 2014;147(3):618-627.e3.

16. Sandborn WJ, Feagan BG, Rutgeerts P, et al. Vedolizumab as induction and maintenance therapy for Crohn's disease. N Engl J Med. 2013;369(8):711-21.

17. Liu GN, Hu SL, Wu JH, et al. China guidelines for pharmacoeconomic evaluation. 2020. China 
Market Press. http://www.scpress.cn/. Accessed 3 Mar 2021. (Chinese-English version)

18. Inflammatory Bowel Disease Group. Chinese consensus on diagnosis and treatment of inflammatory bowel disease (Beijing, 2018). Chin J Pract Intern Med. 2018;38(9):796-813 (In Chinese).

19. Frolkis AD, Dykeman J, Negrón ME, et al. Risk of surgery for inflammatory bowel diseases has decreased over time: a systematic review and metaanalysis of population-based studies. Gastroenterology. 2013;145(5):996-1006.

20. Bodger K, Kikuchi T, Hughes D. Cost-effectiveness of biological therapy for Crohn's disease: Markov cohort analyses incorporating United Kingdom patient-level cost data. Aliment Pharmacol Ther. 2009;30(3):265-74.

21. National Center for Health and Family Planning Commission statistics. Research report on life expectancy and risk factors of Chinese residents (Chinese), 1st edn. Beijing: Peking Union Medical College Press. 2017. http://www.pumcp.com/. Accessed 4 Mar.

22. Lichtenstein GR, Feagan BG, Cohen RD, et al. Serious infection and mortality in patients with Crohn's disease: more than 5 years of follow-up in the TREAT ${ }^{\mathrm{TM}}$ registry. Am J Gastroenterol. 2012;107(9):1409-22.

23. Buxton MJ, Lacey LA, Feagan BG, et al. Mapping from disease-specific measures to utility: an analysis of the relationships between the Inflammatory Bowel Disease Questionnaire and Crohn's Disease Activity Index in Crohn's disease and measures of utility. Value Health. 2007;10(3):214-20.

24. Punekar YS, Hawkins N. Cost-effectiveness of infliximab for the treatment of acute exacerbations of ulcerative colitis. Eur J Health Econ. 2010;11(1): $67-76$.

25. Brown RE, Hutton J, Burrell A. Cost effectiveness of treatment options in advanced breast cancer in the UK. Pharmacoeconomics. 2001;19(11):1091-102.

26. Porco TC, Lewis B, Marseille E, et al. Cost-effectiveness of tuberculosis evaluation and treatment of newly-arrived immigrants. BMC Public Health. 2006;6:157.

27. Hornberger J, Reyes C, Lubeck D, et al. Economic evaluation of rituximab plus cyclophosphamide, vincristine and prednisolone for advanced follicular lymphoma. Leuk Lymphoma. 2008;49(2):227-36.

28. Beusterien KM, Davies J, Leach M, et al. Population preference values for treatment outcomes in chronic lymphocytic leukaemia: a cross-sectional utility study. Health Qual Life Outcomes. 2010;8: 50.

29. Beusterien KM, Szabo SM, Kotapati S, et al. Societal preference values for advanced melanoma health states in the United Kingdom and Australia. Br J Cancer. 2009;101(3):387-9.

30. Vedolizumab Price in China. 2021. https://db. yaozh.com/yaopinzhongbiao?comprehensivesearch content $=\% \mathrm{E} 7 \% \mathrm{BB} \% \mathrm{~B} 4 \% \mathrm{E} 5 \% \mathrm{BE} \% 97 \% \mathrm{E} 5 \% 88 \% \mathrm{~A} \%$ E7\%8F\%A0\%E5\%8D\%95\%E6\%8A\%97\&. Accessed 29 Jan 2021.

31. Scottish Medicine Consortium. Vedolizumab $300 \mathrm{mg}$ powder for concentrate for solution for infusion. SMC No. (1064/15). 2021. https://www. scottishmedicines.org.uk/media/2472/vedolizumab_ entyvio_final_june_2015_for_website.pdf. Accessed 3 Mar 2021. 